\title{
Wireless Sensor Networks Scheduling Algorithm Based on the Probability of Monitoring
}

\author{
Jin-song CHEN \\ Institute of Physics and Electronic Engineering, Yibin University \\ Yibin City, Sichuan Province \\ penglan168@126.com
}

\section{Keywords: Asp.net; E-commerce; Ordering system; Database}

\begin{abstract}
This paper introduces the basics of wireless sensor networks, focusing on the concept of sensor network data collection, data collection mechanisms, data collection in-network data aggregation, data collection and data collection applications Research. This paper presents the data collection in WSN node scheduling concept, analyzed the two data collection in WSN scheduling mechanism, which is based on data collection interval node scheduling mechanism and turn the data based on perceived probability of node sleep scheduling mechanism.
\end{abstract}

\section{Introduction}

Wireless sensor networks (WSN, Wireless Sensor Networks) and wireless sensor networks based on autonomous intelligent system is involved in micro-electromechanical systems, computers, communication, automatic control, artificial intelligence, multi-disciplinary and comprehensive technical. Micro-electromechanical systems (MEMS) laid the rapid development of system on chip design and implementation (SOC) of the base, making the integration of multiple sensor integration, manufacturing, small, low-cost, multi-functional sensor nodes as possible. A large number of MEMS sensor nodes using low-power wireless communication technology connected into a network to play its role in the overall comprehensive; smaller, cheaper, low-power computing devices on behalf of the "post-PC era" to break the traditional desktop computers and high-performance server design patterns, popular network brings immeasurable computing power.

Wireless sensor networks are relatively new areas of international research on wireless sensor networks only a few years. With the development and application of science and technology needs of wireless sensor networks to become one of the hot research in recent years, a large number of wireless sensor network research results appear. However, these findings still in its infancy, still far short of the actual demand. For wireless sensor networks research data collection is not mature, no system, there are a lot of room for improvement.

Radio, infrared, acoustic and other wireless communications technology for the micro-sensor communication between a variety of options, especially in IEEE802.15.4 represented the emergence of short-range wireless communication standard for wireless sensor networks has laid a solid the foundation. At the same time, automatic control and artificial intelligence in swarm intelligence and behavior control of autonomous systems research for the wireless sensor network intelligence to provide strong technical support.

Data collection in wireless sensor networks is one of the main applications, in large sensor networks, a large number of sensor nodes multi-hop wireless ad hoc networks, sensor nodes collect local sensor data, and the results transmitted to the base stations, base station then forwards the data to outside users. In this process, involving many important mechanisms to sensor networks, such as: data collection mechanisms, data collection node scheduling mechanism and data collection in network data aggregation mechanism. The data collection mechanism is an important part of its performance will directly affect the network energy savings, data collection mechanism involves data collection tree construction, data transmission routing, data aggregation mechanism within the network and node scheduling delay and other important content. Therefore, the sensor network data collected by other research study will promote the deepening of the content. 
This paper introduces the basics of wireless sensor networks, focusing on the concept of sensor network data collection, data collection mechanisms, data collection in-network data aggregation, data collection and data collection applications Research.

\section{WSN architecture}

Sensor network consists of a large number of sensor nodes, they are placed in the intensive monitoring of the environment or very close to the measurement environment, nodes without going through the engineering process or pre-positioned, randomly placed in hard to reach terrain or human disaster area, the node running sensor network protocols and algorithms with self-organizing properties. Another feature of the sensor node mutual cooperation between nodes, nodes are equipped with a small processor, so the node is not a large amount of raw data to the cluster nodes, but only the need to address some of the data sent to the sink node.

(1) Hierarchical network communication protocols

Layered network communication protocol from the physical layer, data link layer, network layer, transport layer and application layer.

Physical layer functions, including channel selection, monitoring of the wireless signal, such as sending and receiving signals. Sensor networks using transmission media are radio, infrared, light, etc. The goal is to get as little as possible energy loss larger link capacity. The main physical layer protocols, including narrowband, spread spectrum and ultra wideband technology.

The main task of the data link layer is to strengthen the physical layer transmission function of the original bits, so the network appears as an error-free link [14], which can be divided into media access control (MAC) sub layer and the Logical Link Control (LLC) sub-layer, MAC layer defines how different users share the available channel resources. LLC layer is responsible for providing a unified service interface to the network. The current data link layer of WSN research focused on the MAC layer, MAC protocol and the target for the share from the set of network access channel.

(2) WSN management

Energy management: the sensor network, each node in the power energy is the most valuable resource. Energy management part of the network control node for the use of energy.

Topology Management: In sensor networks, in order to save energy, some node at some point, according to some scheduling rules to sleep, leading to constantly changing network topology, in order to allow the network to normal operation, the need for topology management and control nodes state transition, so the topology management objective is to maintain the smooth flow of the network, data transmission can be effective under the premise of coordination in sensor networks, each node of the state transition.

Network Management: Responsible for network maintenance, diagnostics, network management services provided to the user interface typically includes data collection, data processing, data analysis and troubleshooting functions. Need energy limited WSN, self-organization, and so easily damaged node design of new fully distributed network management system.

QoS support: network and users and between users on the network communicate with each other between the transmission and sharing of information on the quality of the agreement. To meet user requirements, WSN must be able to user applications to provide adequate resources so that they can accept user work performance.

(3) WSN application support technology

WSN application support technology to provide users with a variety of specific application support.

Time synchronization: WSN communication protocols and applications require synchronized clocks at each node. In WSN, the limited capacity of a single node, the system features need to be achieved all the nodes together to complete each other, so time synchronization in WSN nodes coordinated operation plays a very important role. The study included: time server time synchronization algorithm and the dependent channel quality, shorten the synchronization error may cause the "critical path"; from the energy point of view of energy efficient synchronization algorithm. 


\section{WSN node scheduling mechanism for data collection}

Sensor node scheduling is the application layer protocol to study to meet certain coverage, the scheduling of nodes to work at least the state. Sensor nodes densely distributed in the surveillance area, multiple nodes may be the same event monitoring results to the base station, resulting in a large number of redundant data and redundant data processing to increase the network overhead, therefore, on the one hand, the data collection process cited within the network data aggregation mechanism, the compression node of the redundant data collection, reduce redundant data transmission, on the other hand, control the generation of redundant data, application scheduling mechanism to make network data collection cycle in a relatively redundant nodes into sleep state to reduce the generation of redundant data network, saving network energy.

Literature proposed a criterion based on qualified off-duty node scheduling mechanism, when the neighbor nodes can cover a range of sensing, scheduling the sensor nodes to sleep, the mechanism to achieve approximately $100 \%$ coverage, however, the need to estimate the coverage of neighboring nodes, so leading to additional energy consumption by monitoring mechanism to monitor the coverage of the node, mechanisms, one dormant node broadcasts a probe is activated and waiting for a response, if at a given time, the node does not receive a reply message, the node will has remained active, until it runs out of energy, the mechanism does not guarantee the perception of the sensor range and there may be blind spots.

In scheduling, dispatching to the sensing range of sensor nodes and the nodes form a cluster, the cluster of sensor nodes with data collected are interrelated, and therefore, in order to save network energy scheduling cluster nodes take turns to enter the work state. There is a sensor node to set up a cluster, the data collection time is divided into time slices, each cluster node only time the film is in a working state, the remaining nodes in a dormant state, the data collection time for each cycle, the node is active the time (time slice size), if the length of each time slice fixed, different clusters have different data collection period, with cluster size value into a proportional change in the scheduling of a cluster produced, Sink node randomly selected scheduling order, then, the scheduling information is sent to the sensor nodes.

Sensor node is expected to define the perception of coverage (DSC, Desired sensing coverage) for the node coverage and data reporting latency balance between factors that WSN data collection expected quality of service (QoS) level, DSC describes the monitoring area at any point in the node is scheduled to cover the probability ratio, therefore, to meet the requirements of a given DSC under the cover model of adaptive random node selection key scheduling each data collection cycle to determine the number of nodes in the scheduling.

Set up a WSN system consists of a sensor node is identified as identified as the focal point and composition, the sensor nodes are randomly distributed in a monitoring area, the location of nodes and base stations is fixed to predict, monitor the area for each node to collect a certain amount of information, Suppose each node in each time unit generates a data packet transmitted to the base station, the time unit for a reincarnation, network nodes can communicate directly, the information can also be directly transmitted to the base station, each node has a certain energy, base stations have infinite energy supply, each node sends a packet to receive or consume some energy, the energy consumption in data collection is described as. When a node sends out another byte of data points:

$$
\begin{gathered}
E_{T x}(k, d)=E_{\text {elec }}(k)+E_{\text {amp }}(k, d) \\
=\left\{\begin{array}{l}
k E_{\text {elec }}+k \varepsilon_{-f s} d^{2} \\
k E_{\text {elec }}+k \varepsilon_{-m p} d^{4}
\end{array}\right.
\end{gathered}
$$

When a node receives the messages sent, the energy consumption of the wireless receiver.

$$
E_{R x}(k)=k E_{\text {elec }}
$$

The above formula, the wireless transceiver circuit energy consumption, energy consumption of the amplifier, its size depends on the sending node and receiving node and the distance between the bit error rate can be received. When the sending node and receiving node is less than the distance between the threshold value (is a constant, its value depends on the environment), the sender sends 
the data of energy loss is proportional to the square of the distance, or proportional to the fourth power of distance, two energy models are known as free space attenuation model (free space) and multi-channel attenuation model.

Time network described using energy consumption data traffic conditions, the ability to aggregate data set,then copies the data transmission network to transmit an energy reduced the energy consumption data, and network data aggregation with the energy consumed far less than the application of network data aggregation to save energy by.

\section{WSN scheduling algorithm based on the probability of monitoring}

Description of the algorithm. Dense node distribution in wireless sensor networks, how to ensure that areas with large active network monitoring nodes under the conditions of coverage, making the adjacent part of the node turns into active and dormant, while network connectivity is guaranteed to get a better degree of algorithm problem to be solved.

Located in the scheduling cycle $\mathrm{Ti}(\mathrm{t} 1, \mathrm{t} 2, \ldots, \mathrm{tn})$, the active sensor nodes for the $\mathrm{Si}(\mathrm{i}=1,2, \ldots, \mathrm{k})$, node coverage as $\mathrm{Ci}$, monitoring area $\mathrm{A}$, then the scheduling algorithm $\mathrm{S}$ target.

$$
\begin{array}{r}
\mathrm{S}:\left(\left(\bigcup_{\mathrm{i}=1}^{\mathrm{k}} \mathrm{C}_{\mathrm{i}} \bigcap \mathrm{A}\right) \geq \mathrm{A}\right) \bigcap 1\left\{\mathrm{~S}_{\mathrm{i}}, \mathrm{S}_{\mathrm{j}} \notin\left\{\mathrm{t}_{\mathrm{i}}\right\}\right\} \\
\left(\sum \mathrm{x}_{\mathrm{i}}\left(\alpha\left(\delta \mathrm{P}_{\mathrm{i}}+\varphi /\left(\mathrm{A}_{\mathrm{i}} \mathrm{N}_{\mathrm{i}}\right)\right)+\beta\left(\mathrm{U}_{\mathrm{i}}-\mathrm{w}_{\mathrm{i}}\right)\right)\right) \bigcap\left(\sum \mathrm{x}_{\mathrm{i}} \mathrm{w}_{\mathrm{i}} \leq \mathrm{E}\right)
\end{array}
$$

Scheduling algorithm $\mathrm{S}$ for the first phase of the state, the left part of the active nodes that cover close to the maximum degree of coverage monitoring area, right part of that same floor neighbor $\mathrm{Si}$, $\mathrm{Sj} \mathrm{Ti}$ in the scheduling cycle does not choose the same scheduling time slice while ti, that is, if two redundant nodes neighbor each other, it can not go to sleep at the same time, in order to maximize the reduction in monitoring blind spots, while the two adjacent nodes sleep because one of them complement each other to monitor blind spots caused by the relative time will have to be monitored . Meanwhile, the network coverage area to monitor the distribution of different information, so information of different nodes in the region to monitor differences in the probability of active adaptive scheduling time should be adjusted, as energy-efficient scheduling algorithm to improve the accuracy of monitoring information, the same operation residual energy of network nodes in the dynamic changes in this way, adjacent to each other with very similar information to monitor the probability of node residual energy due to the different schedule of its active state the opportunity to be different, in order to balance the energy distribution network nodes, extend the network life-span.

Detailed coverage monitoring scheduling algorithm. Phase One: redundant adjacent nodes choose to schedule.

Completion of phase one monitoring zone node scheduling point selection, inspired by the Sink node, random monitoring of the regional distribution of $\mathrm{k} \mathrm{r}$ a node is equal to scheduling time slice $\mathrm{t}$, $\mathrm{t} 2, \ldots, \mathrm{ti}$, ti values have been relatively small and is continuous the introduction of redundancy backoff algorithm to solve the same layer select the same adjacent node scheduling time slice problem. Network node in the cluster points Sink the incentive, the redundant neighbor nodes exchange information to determine their allocation of time slots scheduling information, if satisfied: (1), the same layer adjacent nodes select the same scheduling time slice. (2) adjacent nodes are selected in different layers of different scheduling time slice. Algorithm is executed.

(1) to determine the basic re-election time, $\Delta \mathrm{t}$ is set.

(2) Define the parameters $\mathrm{k}, \mathrm{k}=\operatorname{Min}[$ reseleck times $\mathrm{m}]$, and is therefore.

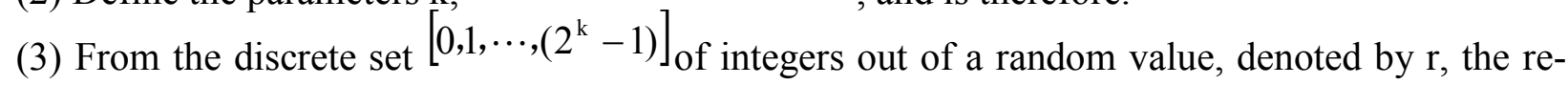
election after the time slice.

(4) When re-election time slice threshold is reached that can not be completed $m$ times the network topology and set the time slice error, to the point of convergence Sink send an error report prompt, otherwise, perform scheduling phase two. 
Stage two: the probability of energy monitoring schedule adjustment

After phase one, the nodes in the network monitoring area to complete the initial dispatch point selection (ti value is relatively small), the scheduling of the monitoring area monitoring node to calculate the probability and energy information to complete the scheduled time to adjust. Network nodes based on data collected, data collection time, the acquisition time collecting data and there are some changes with the application of linear and nonlinear relationships.

$$
\mathrm{y}_{\mathrm{i}}=\sum_{\mathrm{j}=0}^{\mathrm{k}} \beta_{\mathrm{i}} \mathrm{x}_{\mathrm{i}}^{\mathrm{j}}
$$

At that time, 4 is reflected in data collection and acquisition time of the linear relationship between the association model.

$$
\text { Correlation }=\frac{\sum_{i=1}^{n}\left(x_{i}-\bar{x}\right)\left(y_{i}-\bar{y}\right)}{\sqrt{\sum_{i=1}^{n}\left(x_{i}-\bar{x}\right)^{2} \sum\left(y_{i}-\bar{y}\right)^{2}}}
$$

Sensing data and node distribution schedule emerged in the perception of some relevance, if we can calculate the approximate distribution of the data-aware, you can adjust the characteristics of the node distribution of the activation time, therefore, we use the probability of monitoring analysis to adjust the node scheduling, scheduling time to adjust at the same time take into account the energy status of nodes.

We may assume that network initialization, nodes stored in its memory nodes can be used to calculate the probability of part of the monitoring data, sensor nodes based network monitoring probability density function, the classification in its neighborhood in the monitoring of the probability of monitoring area as.

$$
\mathrm{P}\left\{\mathrm{S}_{\mathrm{k}} \mid \mathrm{E} \in \mathrm{C}(\mathrm{i}, \mathrm{j})\right\}=\int_{\mathrm{x} \in C(\mathrm{i}, \mathrm{j})} \mathrm{P}_{\mathrm{s}_{\mathrm{k}}}(\mathrm{x}) \mathrm{dx}
$$

Algorithm analysis and simulation. The introduction of the scheduling algorithm(general scheduling algorithm) and the probability of this scheduling algorithm for the simulation to monitor compares the energy consumption rate based scheduling and data reliability in both the simulation are as follows.

Algorithm is given based on the probability of energy information covering the monitoring node scheduling algorithm is based on data-processing time adaptive scheduling algorithm is reflected in the coverage of connectivity of $\mathrm{S}$ phase of a scheduling algorithm, the initial phase of a complete pre-scheduling, even with randomly distributed network of nodes and its neighbors around the same layer have different scheduling times, but with different layers and their surrounding neighbors to have the same scheduled time because of scheduling in stage one time is relatively small, so that to ensure network coverage in the scheduling, reduce scheduling errors caused by factors in the blind spot, the same neighborhood nodes in different layers of the same scheduling time in scheduling to ensure network connectivity, so the $\mathrm{S}$ phase of a scheduling algorithm to achieve the main function covering the pre-scheduling connected in phase one of the pre-scheduling algorithm based on the scheduling algorithm based on phase II probability of node information and node monitoring maximum residual energy of node scheduling time for adaptation, the scheduling is carried out in a relatively larger period of time in the schedule.

\section{Summary}

This paper describes the data collection in WSN node scheduling concept, analyzed the two data collection in WSN scheduling mechanism, which is based on data collection interval node scheduling mechanism and turn the data based on perceived probability of node sleep scheduling mechanism, the time interval scheduling model, the network node in the data collection period, select 
the appropriate data collection cycle, depending on the chosen time cycle into the active state, perception-based data scheduling mechanism, the node coverage calculation based on data-aware rate, according to the node data-aware rate node sleep probability, according to the probability of scheduling nodes to sleep or work status.

Based on two scheduling mechanisms, this paper a detailed analysis of node-based scheduling mechanism to monitor the probability of energy, the introduction of the mechanism of binary backoff mechanism to solve the node in time slice scheduling conflicts, and to consider the energy balance between nodes, sensor networks in order to effectively achieve data collection node scheduling mechanism, the same time, combined with simulation data node scheduling mechanism to monitor the performance of the probability of the advantages of improved data collection in sensor network node scheduling mechanism.

\section{Acknowledgment}

Campus Youth Fund Project (2011Z14)

\section{References}

[1] M. Ilyas, I. Mahgoub. Handbook of Sensor Networks: Compact Wireless and Wired Sensing System. CRC Press, 2005: 403-419.

[2] RW. Ha, P. H. Ho, X. Sherman Shen, J. Zhang. Sleep scheduling for wireless sensor networks via network flow model. Computer Communications 29 (2006): 2469-2481.

[3]I. Akyildiz, W. Su, Y. Sanakarasubramaniam, et al. Wireless sensor networks: A survey computer Networks. 2002, 38(4): 393-422.

[4] R. Min, M .Bhardwaj, S. Cho, E .Shih, A .Sinha, A .Wang, and A. Chandrakasan. Low--power wireless sensor networks. In Proc. of the 14th International Conference on VLSI Design, Bangalore, India, January 2001. 